\title{
Tubercular Retinal Vasculitis: Diagnostic Dilemma and Management Strategies
}

\author{
Dhananjay Shukla (D) \\ Jay Kalliath ${ }^{2, *}$ \\ Anuradha Dhawan ${ }^{3, *}$ \\ 'Retina- Vitreous Service, Ratan Jyoti \\ Netralaya, Gwalior, India; ${ }^{2}$ Department of \\ Ophthalmology, NMC Specialty Hospital, \\ Abu Dhabi, United Arab Emirates; \\ ${ }^{3}$ Retina- Vitreous Service, Centre for \\ Sight Group of Eye Hospitals, N. Delhi, \\ India
}

*These authors contributed equally to this work

\begin{abstract}
Ocular tuberculosis (OTB) is a rare, extrapulmonary manifestation of systemic $\mathrm{TB}$, which has been a global etiology of uveitis for centuries, though concentrated in the developing world. OTB remains difficult to diagnose clinically despite a plethora of conventional and modern investigations. Tubercular retinal vasculitis (TRV) is a common and specific presentation of OTB but is variably defined in the literature in terms of clinical profile and the investigations essential for diagnosis and treatment. Ironically, the need and duration of antitubercular treatment is uncertain for this manifestation of ocular TB. The rationale and utility for corticosteroids is similarly equivocal for TRV. This review attempts to tease out a commonsense approach from the best available evidence and consensus in the literature to suspect, investigate and diagnose TRV with reasonable certainty, and institute appropriate treatment with due ethnic and geographic considerations.
\end{abstract}

Keywords: ocular tuberculosis, tubercular uveitis, occlusive retinal periphlebitis, tuberculin skin test, chest radiography, antitubercular treatment

\section{Plain Language Summary}

Tuberculosis (TB) is a chronic inflammatory disease, which affects a quarter of the world's population and affects the lungs primarily, though it does not spare any part of the body. TB enters the eye through bloodstream, and therefore the most blood-rich part of the eye, uvea, is most affected (uveitis). For the same reason, the blood vessels commonly get inflamed, primarily the retinal veins: the condition is called tubercular retinal vasculitis (TRV). TB is however difficult to diagnose in the eye, especially TRV, because the inflammation is partly due to bacterial infection, and partly due to allergic reaction to the bacterium. We therefore rely on the typical clinical features of TRV, indirect evidence of tubercular hypersensitivity reaction in the skin and blood, and scars of TB in the lungs on X-ray and CT scan, for a presumptive diagnosis. The antitubercular treatment, which typically lasts half a year, is started on this evidence of exposure to TB. Corticosteroids (strong anti-inflammatory drugs) are added to counter severe inflammation as and when needed. With proactive suspicion and full course of treatment, most cases of TRV can be satisfactorily treated.

\section{Introduction: The Pandemic of Tuberculosis}

Tuberculosis (TB) affects every fourth person on the planet and is the leading cause of death from a single infectious agent worldwide. ${ }^{1}$ Fortunately, most of those affected have asymptomatic latent TB, with only about $5-10 \%$ chance of developing symptomatic TB in their lifetimes. ${ }^{2}$ Although the disease is concentrated in the developing countries, especially in Southeast Asia, the global spread of HIV and the widespread migration of ethnic populations has morphed TB into a pandemic. ${ }^{3}$ The
Correspondence: Dhananjay Shukla Retina-Vitreous Service, Ratan Jyoti Netralaya, Gwalior, 474002, India Tel +917745963548

Email Daksh66@gmail.com 
bacillus Mycobacterium tuberculosis primarily affects the lungs; the extrapulmonary involvement - which represents $16 \%$ of incident cases $^{3}$ - includes bones, intestines, brain, kidneys, genitourinary tract and lymph nodes. Ocular involvement (OTB) is considered an uncommon manifestation of extrapulmonary $\mathrm{TB},{ }^{4}$ incidence ranging from $1.4 \%$ to $18 \%$ according to the endemicity. ${ }^{5}$

\section{The Conundrum of Ocular Tuberculosis}

TB affects the eye mainly via the bloodstream, and consequently the most vascular ocular tissue, uvea, is most commonly affected. ${ }^{5}$ Tubercular uveitis (TBU) is typically a granulomatous inflammation. It may present as anterior, intermediate, posterior uveitis, panuveitis or retinal vasculitis; posterior uveitis is the most common presentation., Tubercular posterior uveitis (choroiditis) could in turn present as serpiginous-like choroiditis, choroidal granuloma or focal/multifocal choroiditis. ${ }^{4,6}$ The variability of clinical picture is only the beginning of the diagnostic riddle. ${ }^{7} \mathrm{~TB}$ is a smoldering low-grade paucibacillary infection which is difficult to culture, more so from the intraocular issue due to inaccessibility, small sample size and inherent risk during culture harvest. ${ }^{8}$ Gene amplification by nested or multiplex polymerase chain reaction (PCR) assay is difficult, expensive, inaccessible in clinical settings, and still unreliable. ${ }^{8}$ Since the most common site of primary involvement are lungs, a chest CT scan or X-ray (CXR, though less reliable is more practical in developing world) may show active or healed lesions of TB. ${ }^{9,10}$ Negative chest imaging however does not rule out ocular TB. ${ }^{6}$ Latent TB can be diagnosed by Tuberculin Skin test (TST) or Interferon-Gamma Release Assay (IGRA), the latter is faster, and does not show false-positive results from BCG vaccination or nonTB mycobacterial infections. ${ }^{2}$ TST scores in low cost, and good predictive value in high-endemic regions. Neither test differentiates active from latent TB. The diagnosis of ocular TB (OTB) is further muddled by the variability of clinical presentations across different ethnic and geographic populations. $^{4-7}$ Collaborative Ocular Tuberculosis Study (COTS) evolved a survey-based consensus of 81 uveitis experts across the globe to clear the murky profile of OTB: the clinically suggestive ocular features when supported by systemic presence of active or latent $\mathrm{TB}$ are sufficient to presumptively diagnose OTB and start antitubercular treatment (ATT). ${ }^{11}$ However, the expert consensus was less clear about the management of anterior or intermediate uveitis and retinal vasculitis. ${ }^{7}$ One reason for the ambiguity was the homogenization of TRV and Eales disease as a single entity, as a manifestation of immune response to the tubercular bacillus.

\section{The Enigma of Tubercular Retinal Vasculitis}

When rheumatologists use the term "vasculitis," they typically refer to a type III hypersensitivity immune reaction where deposition of immune complexes in the medium- or largecaliber vessels leads to vessel wall dissolution and vascular occlusion. ${ }^{12,13}$ When retina or uveitis specialists refer to retinal vasculitis, they refer to perivascular (classically perivenous) inflammatory infiltrates, which are evaluated ophthalmoscopically rather than histopathologically. ${ }^{12-14}$ The terms "vasculitis" and "perivasculitis" are used interchangeably in the context of posterior uveitis, the former term - though less precise - is far more popular due to brevity. Even when ophthalmologists refer to retinal vasculitis, one must look at the regional and ethnic context, which completely changes systemic associations. ${ }^{15}$ Finally, it is pertinent to point out that retinal vasculitis is rarely associated with systemic vasculitis. ${ }^{12}$

There is a dichotomy of opinion in the literature about the when to call a retinal vasculitis isolated, idiopathic or primary, whether to label a primary vasculitis as Eales disease, when to label it secondary (specifically to TB), and finally on the necessity to differentiate between TRV and Eales disease. ${ }^{15-19}$ To begin with the areas of clarity, when retinal vasculitis is associated with choroiditis and active systemic TB, there is no confusion about diagnosis of OTB or TRV. The Collaborative Ocular Tuberculosis Study (COTS-1) study has further clarified that in the presence of specific patterns of choroiditis, serpiginous-like or a tuberculoma, OTB can be labeled merely with association of LTB (positive TST or IGRA). ${ }^{6}$ For TRV, the diagnosis is muddled by the lack of a clear clinical description of retinal vasculitis, which could be labeled tubercular only in the presence of latent TB AND radiological signs of healed $\mathrm{TB}$ in an endemic area, as per COTS study. ${ }^{20-22}$

\section{TRV vs Eales Disease: The Common Ground}

TRV and Eales disease share several common features, which has led to a discussion in uveitis community to either merge the eponym "Eales disease" with TRV or to restrict the former label to a very specific genre of isolated retinal 
vasculitis completely devoid of any ocular or systemic associations, including latent TB. ${ }^{16,17,19,22}$ Indeed, both diseases are diagnoses of exclusion, share the phenotype of occlusive periphlebitis with frequent neovascular complications, are more common in Asian young men with allergic hypersensitivity to tubercular protein as their putative etiopathology, and have displayed a geographic downturn following the recession pattern of TB from the developing countries. ${ }^{15-17}$ Finally, diagnostic studies using skin, serologic PCR-based investigations have shown the evidence of active or latent MTB infection in the etiopathogenesis of the so-called Eales disease. $^{10,16,23,24}$

\section{TRV vs Eales Disease: Chalk and Cheese?}

Despite the aforementioned overlap and ambiguity between the two vasculitic entities, there are several irreconcilable differences between the clinical and therapeutic profiles of TRV and Eales disease. TRV has a specific clinical picture, at least in the regions where most of the cases are reported (Figure 1): it typically presents as an exudative, segmental, hemorrhagic retinal vasculitis, frequently associated with peri- or sub-vascular choroiditis, vitritis, and when more posteriorly situated, with disc and macular edema. ${ }^{22,25,26}$ Eales disease, in contrast, typically presents a peripheral, minimally exudative, nonhemorrhagic, isolated retinal vasculitis with extensive (not segmental) swathes of pipestem-like sheathing; ${ }^{15}$ choroiditis, vitritis, snowball exudates and macular edema are rarely associated. ${ }^{27}$ Eales has the unusual

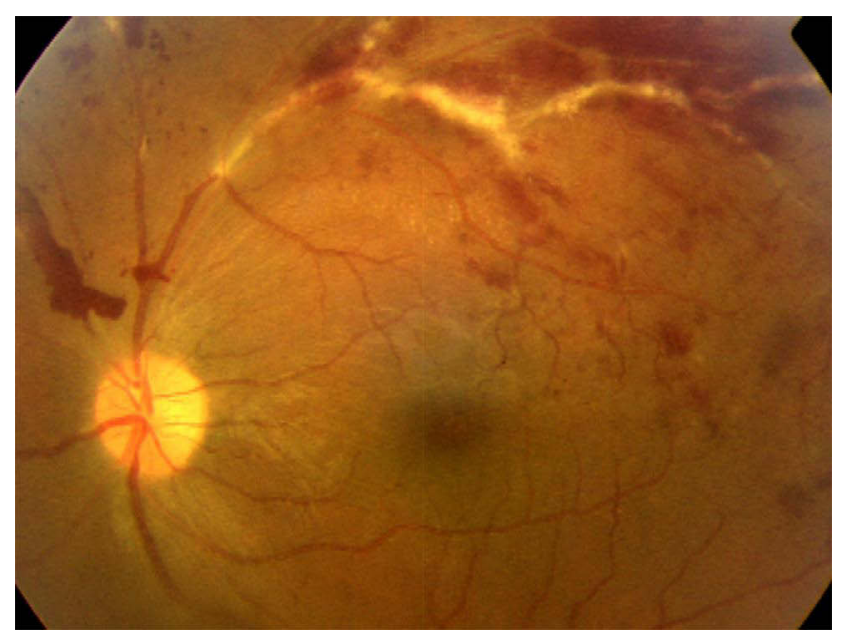

Figure I Active tubercular retinal vasculitis (TRV). Typical fundus appearance of an exudative, hemorrhagic, segmental posterior retinal vasculitis with predominantly venous involvement. Pre-retinal hemorrhage superior-nasal to the optic disc suggests the occlusive nature of vasculitis. This patient had 20/20 Snellen acuity at this time, but macular edema can follow in some patients. combination of minimal inflammation with extensive vascular occlusion causing recurrent vitreous hemorrhages, and sometimes, tractional detachments. ${ }^{15}$ The treatment for Eales is therefore mostly directed to the postinflammation sequelae.

TRV, as the name suggests, is known to be directly caused by Mycobacterium TB bacillus, and therefore has an infective, besides the allergic, component in etiology. It is well known to be treated successfully with ATT alone. ${ }^{26}$ Corticosteroids may indeed be required, but almost never in absence of ATT. A coexistent latent, healed or active systemic TB is mandatory for the diagnosis of TRV; ${ }^{20,22}$ but not for Eales disease, which is a mild primary retinal vasculitis, and does not always require treatment for its inflammatory course. ${ }^{15,27} \mathrm{~A}$ recent study showed that patients with primary retinal vasculitis did not develop TB over a mean follow-up of more than 3 years. ${ }^{18}$ It is noteworthy that this study had a subset labeled as possible TRV (associated with latent TB), based on previously proposed classification of OTB. ${ }^{18,28}$ This subset of possible TRV was possibly the one subsequently alluded to when equating TRV with Eales disease. ${ }^{16,17,20}$ When the anti-inflammatory treatment is required for Eales, systemic or local corticosteroids suffice, ATT is almost never required, in stark contrast to TRV, where ATT is the primary treatment. ${ }^{15,22,25-27}$ These differences in disease presentation, course, and response to treatment compel us to consider TRV and Eales disease as discrete clinical entities.

\section{Natural History of TRV}

Like any retinal vasculitis, TRV courses through the phases of inflammation, occlusion, neovascularization, and tractional complications. The incidence of inflammatory vascular occlusion however varies by geography and ethnicity: it develops in more than $70 \%$ of Asian patients, but in less than $30 \%$ of the White populations. ${ }^{22}$ The consequent peripheral capillary non-perfusion and extraretinal neovascularization (NVE) are therefore common in the Asian phenotype (Figure 2). In a series of 13 PCR positive cases of TRV, development of new vessels (NVD/ NVE) was seen in about $60 \%$ of the eyes after resolution of the vasculitis. ${ }^{29}$ More than half of the patients developed vitreous hemorrhage in a large series of presumed TRV. ${ }^{16}$ Untreated recurrent vitreous bleeds may lead to tractional retinal detachments, iris neovascularization and neovascular glaucoma, highlighting the need for watchful, regular monitoring of these cases. ${ }^{15,18}$ 


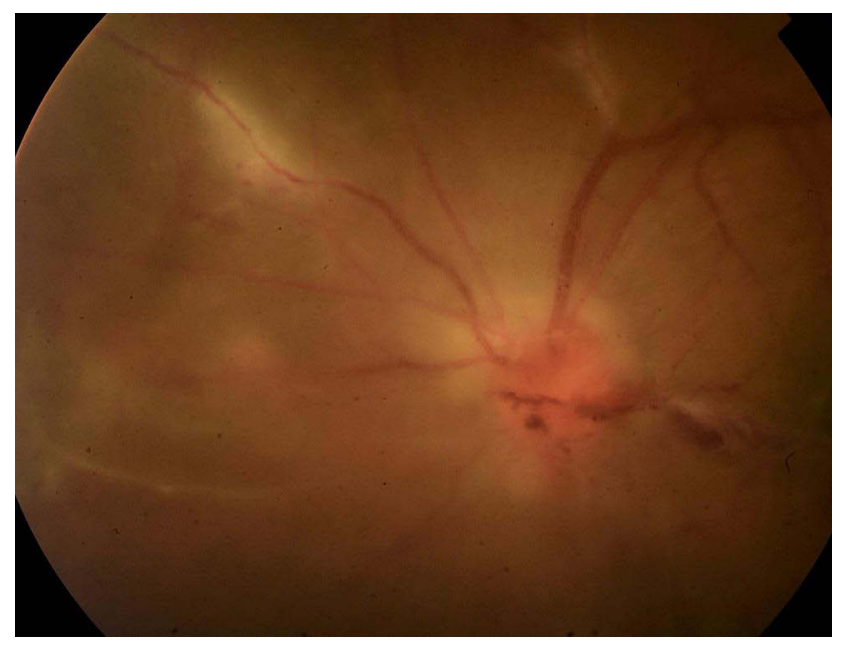

Figure 2 Proliferative stage of TRV. This patient with TRV shows an overlap of inflammatory and proliferative stages of vasculitis: active vasculitis accompanies vitritis, papillitis and vitreous hemorrhage. The key information is that inflammation can directly contribute to neovascularization side-stepping the intervening ischemia. $^{15}$

\section{Indications, Rationale and Modalities of Pharmacotherapy}

Unlike Eales disease, active TRV almost always requires treatment. The treatment is more urgent when the vasculitis is posterior, severe, and symptomatic (associated with macular/disc edema and vitritis). ${ }^{26,29}$ Mild, peripheral TRV in an asymptomatic patient with latent TB from a nonendemic area may not be started on ATT; the patient should however remain under vigilant follow-up of a pulmonologist. ${ }^{16,20}$ Inactive vasculitis, irrespective of the endemicity, should neither be investigated, nor treated (Figure 3); ${ }^{15,16,21}$ the COTS experts did not have consensus on this issue as there is no ocular or systemic endpoint for ATT in this situation. ${ }^{20}$

Once the diagnosis of TRV is established based on phenotype (see above), positive immunologic AND radiologic investigations, the treatment with ATT is a given, ${ }^{20}$ since healed pulmonary $\mathrm{TB}$ is a risk factor for conversion of latent to active systemic TB. ${ }^{2}$ ATT is also essential to prevent recurrences of OTB. ${ }^{29}$ In the presence of active systemic TB, a full course of ATT is also a no-brainer (though active TB is rarely found to be associated with OTB). However, the COTS-2 survey did not reach consensus on how to administer ATT for systemically inactive (healed lesions on $\mathrm{CXR} / \mathrm{CT}$ ) or latent TB: full 6, 9 or 12-month therapy starting with 4 drugs, or ATT toned down for latent TB. ${ }^{11,19,30}$ The duration varied according to the severity of OTB, endemicity of TB, other high-risk features (like healed lung

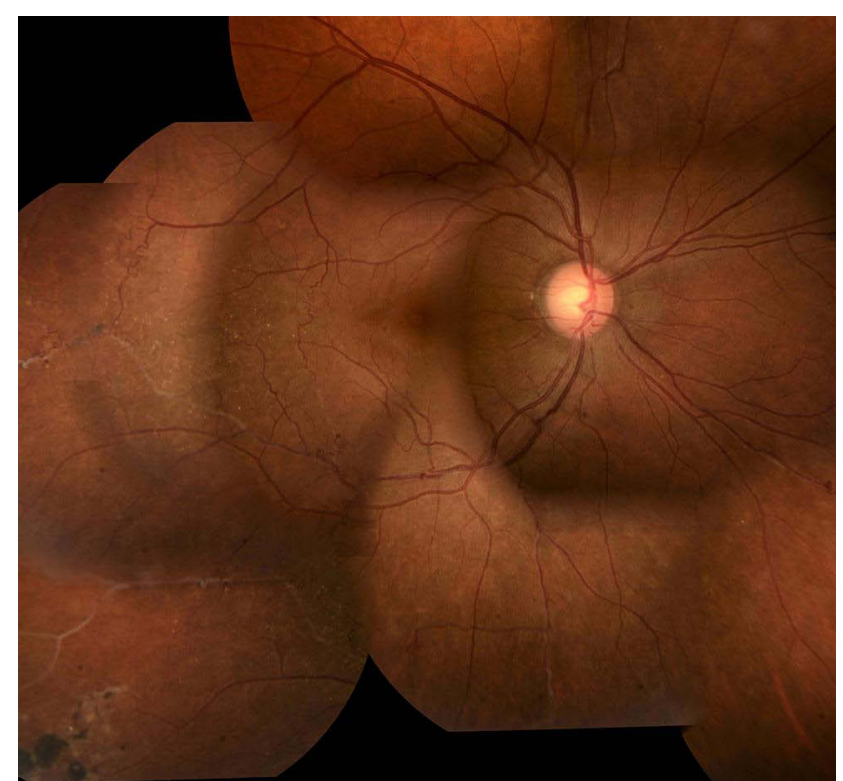

Figure 3 Inactive retinal vasculitis. This patient has 20/20 Snellen acuity with regressed retinal vasculitis with sheathed vessels. Note a chorioretinal atrophic patch with pigment clumps secondary to healed chorioretinitis inferotemporally. There are some collaterals around the temporal vascular arcades indicating compensated retinal ischemia. Such patients need only be observed and counseled for regular follow-ups. Ocular (angiography) or systemic (serologic/radiologic) investigations are not required.

lesions, HIV) and initial response to ATT. The international guidelines suggest a 6-month trial as the default regimen in view of the paucibacillary latent infection in most cases. ${ }^{31}$

The COTS studies were also equivocal about the fundus phenotype of TRV to qualify for ATT, ${ }^{20-22}$ which could lead to overdiagnosis of OTB in high-endemic countries like India, a possibility accepted by COTS investigators. ${ }^{11,21,30}$ This overdiagnosis could be expensive as toxicity of ATT is well recognized but was not documented in the COTS study. ${ }^{30}$ One clear way to avoid overdiagnosis as well as missed treatment is to recognize the clinical features of vasculitis suggestive of OTB and reserve ATT for them. ${ }^{25,26}$ Equivocal diagnostic criteria for TRV were the reason the largest multinational study on TRV was unable to show any significant therapeutic effect of ATT. $^{22}$ Likewise, a cohort study on isolated retinal vasculitis with features of presumed TRV also failed to show any benefit of ATT, or late appearance of systemic TB over a long follow-up. ${ }^{18}$ The lack of attention on diagnostic clinical profile of TRV appeared to be the common ground for inexplicable treatment outcomes like COTS: one should not label retinal vasculitis as tubercular if ATT was of questionable benefit. ${ }^{20-22}$ 
The second key component of pharmacotherapy is corticosteroids. Two specific manifestations of OTB, serpiginouslike choroiditis and TRV, are probably apt candidates for corticosteroids since both are likely to have a significant immunologic component in their etiology. ${ }^{4}$ In the context of TRV, corticosteroids may be required when the inflammation is sight-threatening: posterior, severe, hemorrhagic vasculitis with vitritis and macular edema, which probably indicates an added immune response to bacterial infection. ${ }^{26}$ Another indication for steroids in OTB is in cases of paradoxical worsening due to exaggerated inflammatory response after ATT. Though COTS study had the largest collection of TRV cases so far, because of ambiguity about definition, it could not clearly define the role of corticosteroids: whether they were beneficial, redundant, or harmful in routine treatment of $\mathrm{TRV}^{22}$ One reason was that steroids were used by default in almost all of the cases. ${ }^{11}$ A rationale for high frequency of corticosteroid usage could be the predominantly occlusive nature of vasculitis - which required early control to prevent ischemic complications - whether secondary to TB or other causes like Behcet's disease. ${ }^{17}$ A recent controlled study however showed that for TRV sans the previously mentioned high-risk features, ATT alone suffices to treat the inflammation completely, and without recurrences. ${ }^{26} \mathrm{~A}$ meta-analysis of 28 clinical studies also failed to show any benefit of concomitant systemic steroids on the treatment outcomes, probably because the rationale for their use was not specified. ${ }^{31}$ To avoid the systemic sideeffects of steroids, local therapy in the form of intravitreal dexamethasone implants can be used in cases of corticosteroid intolerance, especially when TRV is unilateral. ${ }^{32}$

\section{Management of Neovascular Sequelae and Complications}

Remission of inflammation is not the end of the story for TRV. As a sequel to inflammatory vascular occlusions, extensive capillary dropouts develop; consequent extraretinal neovascularization requires peripheral sectoral or scatter retinal photocoagulation for the ischemic areas as delineated by fluorescein angiography (Figure 4) ${ }^{15}$ Newer technologies like ultra-wide field angiography are helpful in mapping the entire extent of peripheral retinal ischemia for better coverage with photocoagulation. ${ }^{33}$ Optic disc neovascularization generally indicates more widespread ischemia requiring panretinal photocoagulation. ${ }^{15}$ Photocoagulation is contraindicated in the presence of active vasculitis, which can be detected by fluorescein angiography in equivocal cases (Figure 5; Table 1),

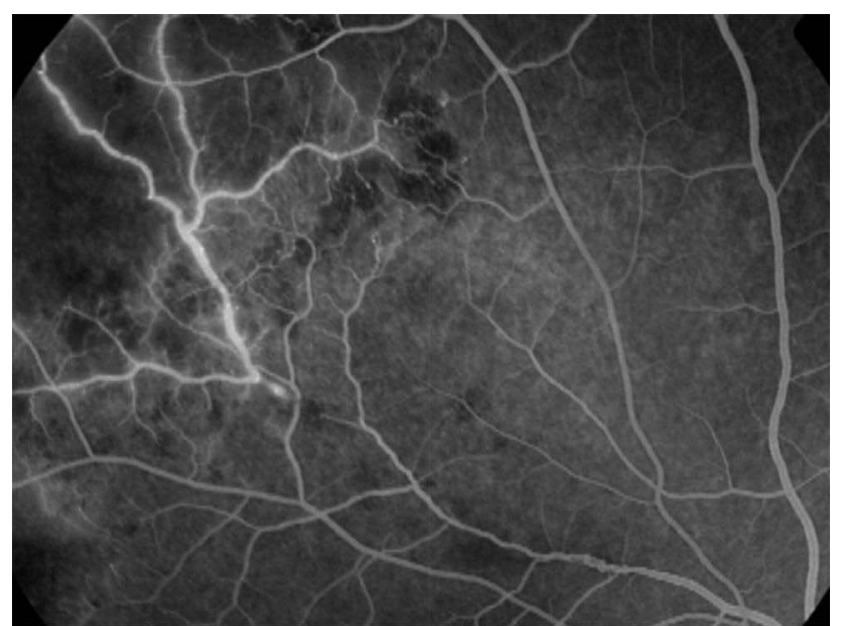

Figure 4 Fluorescein angiography showing inactive TRV. Like Figure 3 (not the same eye), this angiogram shows peripheral capillary non-perfusion in the left upper corner; the blocked fluorescence along the proximal edge of non-perfusion is due to blot hemorrhages and appears darker. Note that the venules draining the capillary dropout are stained, but not leaking: this appearance is suggestive of inactive sheathing, as shown in Figure 3.

and is likely to flare up and release more angiogenic factors, aggravating neovascularization. ${ }^{15,29}$

Vitrectomy is indicated in tubercular or other occlusive vasculitis for visually significant epiretinal membrane or vitreomacular traction, non-clearing vitreous hemorrhage, persistent vitritis despite medical management, progressive tractional retinal detachment and finally for diagnostic biopsy in intractable cases. ${ }^{15,17,34-37}$ Tractional and combined-mechanism detachments are the most complex surgical indications in retinal vasculitis, which may limit the

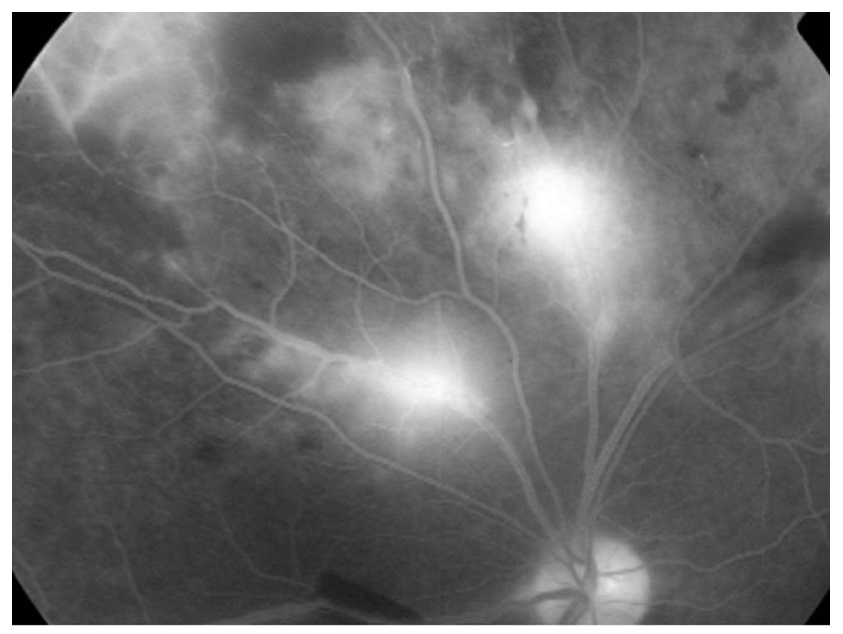

Figure 5 Fluorescein angiography showing active TRV. In contrast to Figure 4, active vasculitis shows leakage from the vessel wall: more intense from the venules proximal to the optic nerve head, and less intense from the peripheral venule in the left upper corner, but with adjoining capillary nonperfusion. As the active vasculitis regresses, the occlusive phase starts from periphery in the same eye. 
Table I Diagnostic Work-up of Tubercular Retinal Vasculitis (TRV)

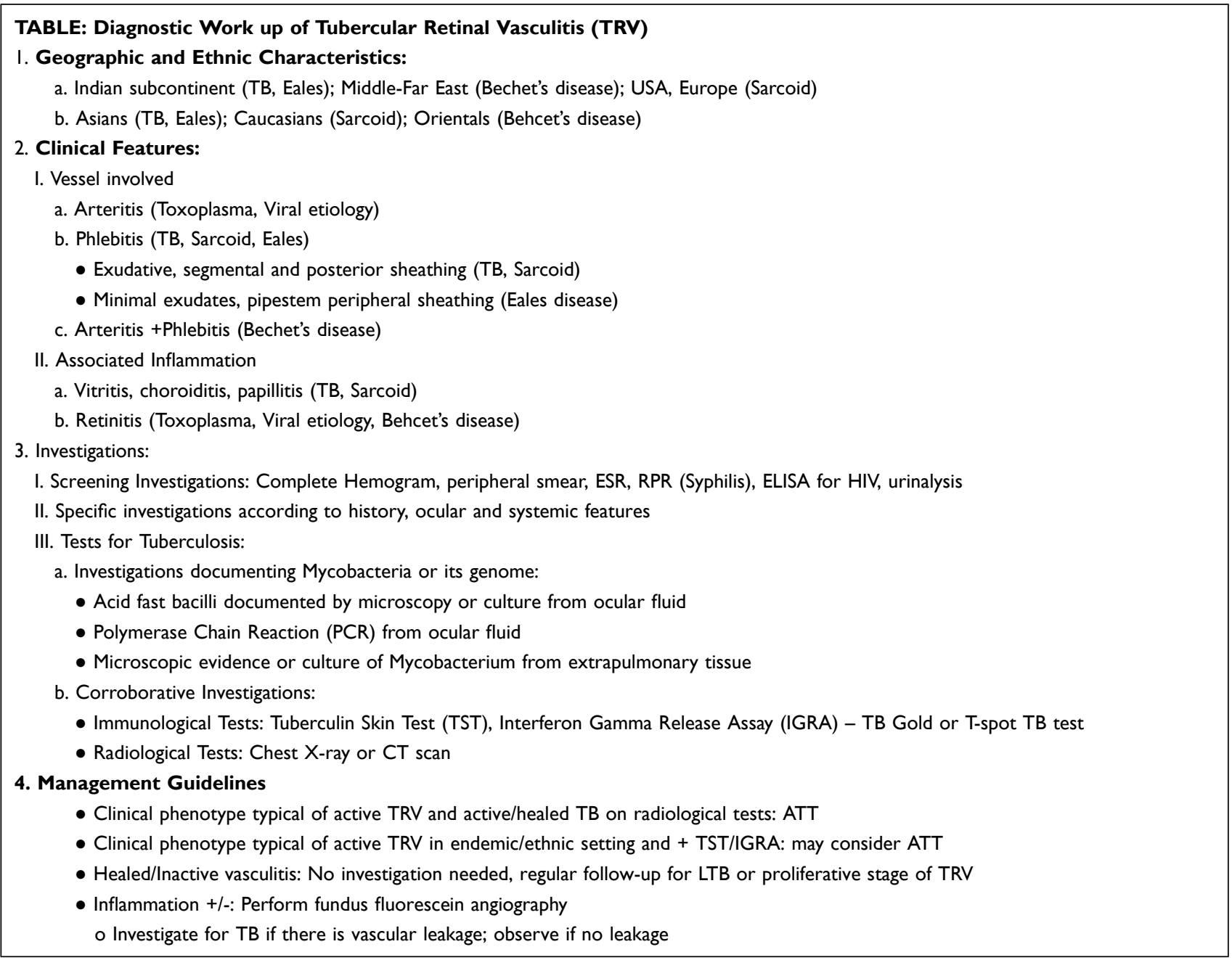

Abbreviations: TB, tuberculosis; ESR, erythrocyte sedimentation rate; RPR, rapid plasma reagin test; ELISA, enzyme-linked immunosorbent assay; HIV, human immunodeficiency viruses; ATT, antitubercular treatment; LTB, latent tuberculosis.

functional gains in spite of good anatomical outcomes. ${ }^{34,35}$ Vitrectomy even in the absence of tractional detachment could be complicated by tightly adherent peripheral membranes with thin ischemic underlying retina, vitreoschisis, and difficult induction of posterior vitreous detachment. ${ }^{34}$ In young patients with clear lens, an encircling belt buckle might help as peripheral membranes could be difficult to access. ${ }^{34,35}$ Vitrectomy can also help in faster resolution of uveitis by the removal of inflammatory debris from the vitreous. ${ }^{36}$ It could also be a diagnostic aid in patients with inconclusive TST/QFT and chest radiography by providing a vitreous sample for molecular diagnosis (PCR). ${ }^{37}$

\section{Summing Up the Clarity on Evidence and Consensus}

Three centuries of familiarity with the tubercular uveitis and several clinical, laboratory and technological innovations later, we have still not been able to unravel the mystery of diagnosis and treatment of OTB, mainly due to the paucibacillary nature of disease, wide range of uveitis presentations and variable extent of immune-mediated response in the eye. ${ }^{7,30}$ It is pertinent to note that OTB is not exclusively a developing world problem: a tertiary clinic in the USA reported an average delay of more than 2 years in the diagnosis of OTB, resulting in profound visual loss in about $40 \%$ of the patients. ${ }^{38}$ TRV remains a particularly elusive subset of OTB with even more disputed management. ${ }^{20,21,26}$ A commonsense approach can however be distilled out from the literature to guide the treating ophthalmologist (Table 1). Retinal vasculitis conveys different etiopathologic connotations according to geography and ethnicity: TRV should be suspected primarily in areas endemic for latent TB like the Indian subcontinent, and in vulnerable ethnic groups like Asians, Middle-Easterns and Africans. ${ }^{15,22}$ The 
suspicion is augmented in presence of classic but not pathognomonic fundus features, which require ruling out other systemic infections and inflammations like sarcoidosis, syphilis, Behcet's disease etc. by detailed history, systemic examination and tailored serologic, immunologic and radiologic investigations. ${ }^{11,15,25,26,39}$ In the presence of a clinical picture suggestive of TRV, detection of latent and healed TB is sufficient to start ATT; molecular/ histopathologic diagnosis is an impractical gold standard. ${ }^{4,6,20,31}$ If the vasculitis is not severe and sight-threatening, ATT alone may suffice to treat if fully. ${ }^{26}$ Corticosteroids, when required for sightthreatening conditions, should start and stop under ATT cover, ${ }^{11}$ to tide over the acute inflammation without aggravating infection. The standard 6-month duration of ATT is enough in most cases, as the associated systemic TB is typically latent or inactive. ${ }^{31}$ Finally, the absence of response to ATT in 2-3 months should alert the ophthalmologist to the possibility of refractory TB or alternative diagnoses. ${ }^{4,31}$ TRV remains a formidable yet unavoidable clinical challenge for the ophthalmologists worldwide. However, with due attention to history, ocular findings, systemic status and tailored investigations, the diagnosis can be reached with reasonable certainty in most cases. Subsequent pharmacotherapy, photocoagulation or vitrectomy usually deliver rewarding outcomes for the patient and the physician.

\section{Disclosure}

The authors report no financial interest or conflicts of interest in this work.

\section{References}

1. World Health Organization [homepage on internet]. Tuberculosis. Available from: https://www.who.int/news-room/fact-sheets/detail/ tuberculosis. Accessed September 15, 2021.

2. Rajpal S, Arora VK. Latent TB (LTBI) treatment: challenges in India with an eye on 2025: "To Treat LTBI or not to treat, that is the question". Indian J Tuberc. 2020;67(4S):S43-S47. doi:10.1016/j. ijtb.2020.09.028

3. World Health Organization [homepage on internet]. Global tuberculosis report; 2020. Available from: https://www.who.int/teams/globaltuberculosis-programme/tb-reports/global-tuberculosis-report-2020. Accessed September 15, 2021.

4. Betzler BK, Gupta V, Agrawal R. Clinics of ocular tuberculosis: a review. Clin Exp Ophthalmol. 2021;49(2):146-160. doi:10.1111/ ceo. 13847

5. Dalvin LA, Smith WM. Intraocular manifestations of mycobacterium tuberculosis: a review of the literature. J Clin Tuberc Other Mycobact Dis. 2017;7:13-21. doi:10.1016/j.jctube.2017.01.003

6. Agrawal R, Testi I, Mahajan S, et al. Collaborative ocular tuberculosis study consensus guidelines on the management of tubercular Uveitis-Report 1. Ophthalmology. 2021;128(2):266-276. doi:10.1016/j.ophtha.2020.01.008
7. Rosenbaum JT, Van Gelder RN. Tautologies and the Telltale Tubercle Bacillus. Ophthalmology. 2021;128(2):288-289. doi:10.1016/j. ophtha.2020.11.005

8. Wroblewski KJ, Hidayat AA, Neafie RC, Rao NA, Zapor M. Ocular tuberculosis: a clinicopathologic and molecular study. Ophthalmology. 2011;118(4):772-777. doi:10.1016/j.ophtha.20 10.08.011

9. Bansal R, Gupta A, Agarwal R, et al. Role of CT chest and cytology in differentiating tuberculosis from presumed sarcoidosis in uveitis. Ocul Immunol Inflamm. 2019;27(7):1041-1048. doi:10.1080/ 09273948.2018.1425460

10. Kharel Sitaula R, Iyer V, Noronha V, Dutta Majumder P, Biswas J. Role of high-resolution computerized tomography chest in identifying tubercular etiology in patients diagnosed as Eales' disease. J Ophthalmic Inflamm Infect. 2017;7(1):4. doi:10.1186/s12348-0160120-1

11. Agrawal R, Gunasekeran DV, Grant R, et al. Clinical features and outcomes of patients with Tubercular Uveitis treated with antitubercular therapy in the Collaborative Ocular Tuberculosis Study (COTS)-1 [published correction appears in JAMA Ophthalmol. 2019 Feb 1;137(2):233]. JAMA Ophthalmol. 2017;135 (12):1318-1327. doi:10.1001/jamaophthalmol.2017.4485

12. Rosenbaum JT, Sibley CH, Lin P. Retinal vasculitis. Curr Opin Rheumatol. 2016;28(3):228-235. doi:10.1097/BOR.0000000 000000271

13. Graham EM, Stanford MR, Whitcup SM. Retinal vasculitis. In: Pepose JS, Holland GN, Wilhelmus KR, editors. Ocular Infection and Immunity. St. Louis: Mosby; 1996:538-551.

14. El-Asrar AM, Herbort CP, Tabbara KF. A clinical approach to the diagnosis of retinal vasculitis. Int Ophthalmol. 2010;30(2):149-173. doi:10.1007/s10792-009-9301-3

15. Shukla D, Namperumalsamy P. Eales Disease. In: Schachat AP, Wilkinson CP, Hinton DR, Wiedemann P, editors. Ryan's Retina. 6th ed. Edinburgh; New York: Elsevier; 2018:1665-1670.

16. Agrawal R, Gunasekeran DV, Gonzalez-Lopez JJ, et al. Peripheral retinal vasculitis: analysis of 110 consecutive cases and a contemporary reappraisal of tubercular etiology. Retina. 2017;37 (1):112-117. doi:10.1097/IAE.0000000000001239

17. Agarwal A, Karkhur S, Aggarwal K, et al. Epidemiology and clinical features of inflammatory retinal vascular occlusions: pooled data from two tertiary-referral institutions. Clin Exp Ophthalmol. 2018;46(1):62-74. doi:10.1111/ceo.12997

18. Kawali A, Bavaharan B, Sanjay S, Mohan A, Mahendradas P, Shetty B. A long-term follow-up of retinal vasculitis - Do they develop systemic disease? Ocul Immunol Inflamm. 2020;28 (8):1181-1186. doi:10.1080/09273948.2019.1697455

19. Petrushkin H, Sethi C, Potter J, et al. Developing a pathway for the diagnosis and management of ocular tuberculosis. The pan-London ocular tuberculosis pathway-LOOP. Eye. 2020;34(5):805-808. doi:10.1038/s41433-019-0543-7

20. Agrawal R, Testi I, Bodaghi B, et al. Collaborative ocular tuberculosis study consensus guidelines on the management of Tubercular Uveitis-Report 2: guidelines for Initiating antitubercular therapy in anterior uveitis, intermediate uveitis, panuveitis, and retinal vasculitis. Ophthalmology. 2021;128(2):277-287. doi:10.1016/j. ophtha.2020.06.052

21. Shukla D. Re: agrawal et al.: collaborative ocular tuberculosis study consensus guidelines on the management of tubercular uveitis Report 2: guidelines for initiating antitubercular therapy in anterior uveitis, intermediate uveitis, panuveitis, and retinal vasculitis (Ophthalmology. 2021;128:277-287). Ophthalmology. 2021;128(7): e34-e35. doi:10.1016/j.ophtha.2021.03.024

22. Gunasekeran DV, Agrawal R, Agarwal A, et al. The Collaborative Ocular Tuberculosis Study (COTS)-1: a multinational review of 251 patients with tubercular retinal vasculitis. Retina. 2019;39 (8):1623-1630. doi:10.1097/IAE.0000000000002194 
23. Singh R, Toor P, Parchand S, Sharma K, Gupta V, Gupta A. Quantitative polymerase chain reaction for Mycobacterium tuberculosis in so-called Eales' disease. Ocul Immunol Inflamm. 2012;20 (3):153-157. doi:10.3109/09273948.2012.658134

24. Bansal R, Moharana B, Katoch D, Gupta V, Dogra MR, Gupta A. Outcome of pars plana vitrectomy in patients with retinal detachments secondary to retinal vasculitis. Indian J Ophthalmol. 2020;68 (9):1905-1911. doi:10.4103/ijo.IJO_551_20

25. Kaza H, Modi R, Rana R, et al. Effect of adjunctive pars plana vitrectomy on focal posterior segment inflammation: a case-control study in tuberculosis-associated uveitis. Ophthalmol Retina. 2018;2 (11):1163-1169. doi:10.1016/j.oret.2018.05.002

26. Bansal R, Dogra M, Chawla R, Kumar A. Pars plana vitrectomy in uveitis in the era of microincision vitreous surgery. Indian J Ophthalmol. 2020;68(9):1844-1851. doi:10.4103/ijo.IJO_1625_20

27. Patel SS, Saraiya NV, Tessler HH, Goldstein DA. Mycobacterial ocular inflammation: delay in diagnosis and other factors impacting morbidity. JAMA Ophthalmol. 2013;131(6):752-758. doi:10.1001/ jamaophthalmol.2013.71

28. George RK, Walton RC, Whitcup SM, Nussenblatt RB. Primary retinal vasculitis. Systemic associations and diagnostic evaluation. Ophthalmology. 1996;103(3):384-389. doi:10.1016/s0161-6420(96) 30681-7

29. Gupta P, Biswas J. Further evidence of the association of latent Mycobacterium tuberculosis in Eales' disease. Int Ophthalmol. 2021;41(3):901-906. doi:10.1007/s10792-020-01645-5

30. Kaza H, Tyagi M, Pathengay A, Basu S. Clinical predictors of tubercular retinal vasculitis in a high-endemic country. Retina. 2021;41(2):438-444. doi:10.1097/IAE.0000000000002829
31. Kelgaonkar A, Govindhari V, Khalsa A, Basu S. Anti-tubercular therapy alone for treatment of isolated tubercular retinal vasculitis [published online ahead of print, 2021 Aug 9]. Eye. 2021. doi:10.1038/s41433-021-01727-3

32. Biswas J, Reesha KR, Pal B, Gondhale HP, Kharel Sitaula R. Long-Term outcomes of a large cohort of patients with Eales' disease. Ocul Immunol Inflamm. 2018;26(6):870-876. doi:10.1080/09273948.2017.1298817

33. Gupta A, Sharma A, Bansal R, Sharma K. Classification of intraocular tuberculosis. Ocul Immunol Inflamm. 2015;23(1):7-13. doi: 10.3109/09273948.2014.967358

34. Gupta A, Gupta V, Arora S, Dogra MR, Bambery P. PCR-positive tubercular retinal vasculitis: clinical characteristics and management. Retina. 2001;21(5):435-444. doi:10.1097/00006982-200110000-00004

35. Van Gelder RN. Uveitis-the tortured tale of the tubercle. JAMA Ophthalmol. 2017;135(12):1328-1329. doi:10.1001/jamaopht halmol.2017.3514

36. Kee AR, Gonzalez-Lopez JJ, Al-Hity A, et al. Anti-tubercular therapy for intraocular tuberculosis: a systematic review and meta-analysis. Surv Ophthalmol. 2016;61(5):628-653. doi:10.1016/ j.survophthal.2016.03.001

37. Agarwal A, Handa S, Aggarwal K, et al. The role of dexamethasone implant in the management of Tubercular Uveitis. Ocul Immunol Inflamm. 2018;26(6):884-892. doi:10.1080/09273948.2017.1400074

38. Jones NP, Sala-Puigdollers A, Stanga PE. Ultra-widefield fundus fluorescein angiography in the diagnosis and management of retinal vasculitis. Eye. 2017;31(11):1546-1549. doi:10.1038/eye.2017.93

39. Shukla D, Kanungo S, Prasad NM, Kim R. Surgical outcomes for vitrectomy in Eales' disease. Eye. 2008;22(7):900-904. doi:10.1038/ sj.eye. 6702763
Clinical Ophthalmology

\section{Publish your work in this journal}

Clinical Ophthalmology is an international, peer-reviewed journal covering all subspecialties within ophthalmology. Key topics include: Optometry; Visual science; Pharmacology and drug therapy in eye diseases; Basic Sciences; Primary and Secondary eye care; Patient Safety and Quality of Care Improvements. This journal is indexed on PubMed

\section{Dovepress}

Central and CAS, and is the official journal of The Society of Clinical Ophthalmology (SCO). The manuscript management system is completely online and includes a very quick and fair peer-review system, which is all easy to use. Visit http://www.dovepress.com/ testimonials.php to read real quotes from published authors. 